\title{
EXTENSION OF AUTOCOVARIANCE COEFFICIENTS SEQUENCE FOR PERIODICALLY CORRELATED PROCESSES
}

\author{
By Sophie LAMBert-Lacroix
}

Laboratoire LMC-IMAG

Final Version received September 2003

\begin{abstract}
The extension of stationary process autocorrelation coefficient sequence is a classical problem in the field of spectral estimation. In this note, we treat this extension problem for the periodically correlated processes by using the partial autocorrelation function. We show that the theory of the non-stationary processes can be adapted to the periodically correlated processes. The partial autocorrelation function has a clear advantage for parameterization over the autocovariance function which should be checked for non-negative definiteness. In this way, we show that contrary to the stationary case, the Yule-Walker equations (for a periodically correlated process) is no longer a tool for extending the first autocovariance coefficients to an autocovariance function. Next, we treat the extension problem and present a maximum entropy method extension through the the partial autocorrelation function. We show that the solution maximizing the entropy is a periodic autoregressive process and compare this approach with others.
\end{abstract}

Keywords. Maximum entropy method; partial autocorrelation; periodically correlated processes; periodic autoregressive processes.

\section{INTRODUCTION}

The class of periodically correlated (PC) processes, introduced by Gladysev (1961), is useful in many signal processing problems (e.g. Gardner, 1994, and references therein). They are of interest with regard to multivariate stationary processes. They also provide a deeper insight into these processes and facilitate their modelling.

Although the partial correlation notion was introduced years ago by Yule (1907), the parameterization of a stationary time series by the partial autocorrelation function (PACF) is relatively recent (Ramsey, 1974). This result has been established by Dégerine and Lambert-Lacroix (2003) (see also Constantinescu, 1996) to the general non-stationary case. The PACF presents many advantages when compared with the classical autocovariance function (ACF). For example, the simplicity of the constraints on the PACF gave birth to many autoregressive estimation methods in the stationary framework (Burg, 1968; Dégerine, 1993) and PC (Sakai, 1982; Boshnakov, 1994; Lambert-Lacroix, 2000).

In the stationary case, the extension problem of autocovariance coefficient sequence can be stated as follows. Starting from $p+1$ coefficients $R(0), \ldots, R(p)$ 
(i.e. the possible covariance structure of a sequence $X(t), \ldots, X(t-p)$ ), we can say whether the values are the first coefficients of an ACF and thereby describe all the extensions. For this purpose, it is better to use the PACF because of the simplicity of its constraints domain. Furthermore, the maximum entropy method (MEM), first introduced by Burg (1967), consists in extending $R(0), \ldots, R(p)$, in such a way it maximizes the entropy of the stationary random vector $X(t), \ldots, X(t-n), n>p$. It turns out that the only such extension is given by the Yule-Walker equations and the solution is an autoregressive model.

In this note, we treat this extension problem for PC processes by using the PACF. First we show that the theory of the non-stationary processes can be adapted to the periodical case. In particular, the PACF is periodic as the ACF. In the stationary case, we extend the ACF to lags $0,1, \ldots, p$. Similarly, in the periodic case, it is natural to consider extending ACF (or PACF) to lags $0,1, \ldots, p_{k}, k=$ $1, \ldots, T$ (i.e. $p_{t}$ lags for the season $t$ ). We show that the correspondence between ACF and PACF is one-to-one if and only if $p_{t} \leq p_{t-1}+1, p_{0}=p_{T}$. As in the stationary case, the PACF coefficients are between -1 and 1 . This has a clear advantage for parameterization over the ACF which should be checked for nonnegative definiteness. Contrary to the stationary framework, we show that it is not always possible to fit a PAR of given orders $\left(p_{1}, \ldots, p_{T}\right)$. We propose a procedure which allows to check (for ACF coefficients and orders given) the existence of the PAR model. As a consequence, the Yule-Walker equations (for a PC process) do no longer provide a tool to extend the first ACF coefficients to the ACF of a PC process. Next, we treat the extension problem in the periodical case. Finally, we present a MEM extension through the PACF. We show that the solution maximizing the entropy is periodic autoregressive. This approach is compared with those of Zhang (1997) and Alpay et al. (2001).

\section{PERIODICALLY CORRELATED PROCESSES}

\subsection{Parameterization by the $P A C F$}

First, let us state some preliminary known results (see Lambert-Lacroix, 2000; Dégerine and Lambert-Lacroix, 2003; for more comments). The PC processes $X(\cdot)$, of period $T$, indexed on $\mathbb{Z}$ with $E(X(t))=0$ for any $t \in \mathbb{Z}$, were introduced by Gladysev (1961) as non-stationary processes with periodic ACF,

$$
R(t+T, s+T)=E\{X(t+T) \overline{X(s+T)}\}=R(t, s) \text { for all }(t, s) \in \mathbb{Z}^{2},
$$

where - denotes the complex conjugate. In other words, the second-order properties of these processes are left unchanged by time translation of length $T$. The structure of $\{X(s+T), \ldots, X(t+T)\}$ and that of $\{X(s), \ldots, X(t)\}$ are the same. This function must be non-negative definite (n.n.d.), i.e.

$$
R_{s, t}=\{R(s+i, s+j)\}_{i, j=0, \ldots, t-s}
$$


is n.n.d for all $s \leq t$ (as the covariance matrix of the random vector $\left.[X(s), \ldots, X(t)]^{\mathrm{T}}\right)$.

Let us now recall the definition of the PACF. In this paper, we only deal with the second-order properties of the process $X(\cdot)$. So it is natural to use a geometrical approach by considering the following Hilbert space $\mathcal{M}$, with the Hermitian product $\langle U, V\rangle=E\{U \bar{V}\}=\operatorname{cov}\{U, V\}$. The elements of $\mathcal{M}$ are the linear combinations, with complex coefficients, of elements of $\{X(t), t \in \mathbb{Z}\}$ and their mean square limits. Let $X^{f}(t ; s), s \leq t$, be the orthogonal projection of $X(t)$ on the closed linear subspace

$$
\mathcal{M}(s ; t-1)=\overline{s p}\{X(s), \ldots, X(t-1)\},
$$

that is, the linear predictor of $X(t)$ given $X(t-1), \ldots, X(s)$, with the convention $X^{f}(t ; t)=\overrightarrow{0}$. The $(t-s)$ th-order forward partial innovation is $\varepsilon^{f}(t ; s)=$ $X(t)-X^{f}(t ; s)$. We put

$$
\sigma^{2 f}(t ; s)=\left\|\varepsilon_{\varepsilon}^{f}(t ; s)\right\|^{2}=\operatorname{var}\left\{\varepsilon^{f}(t ; s)\right\}
$$

The associated normalized innovation is defined, for $s \leq t$, by

$$
\eta^{f}(t ; s)=\frac{\varepsilon^{f}(t ; s)}{\sigma^{f}(t ; s)}, \quad \sigma^{f}(t ; s)=\sqrt{\sigma^{2 f}(t ; s)} ;
$$

with the convention $0^{-1}=0$. Note that this convention is necessary in the locally deterministic case when $X(t) \in \mathcal{M}(s ; t-1)$. All the notions obtained by reversing the time index are denoted by ' $b$ ' for 'backward'; for instance, for $s \leq t$, the backward innovation $\varepsilon^{b}(s ; t)=X(s)-X^{b}(s ; t)$ leads to

$$
\eta^{b}(s ; t)=\frac{\varepsilon^{b}(s ; t)}{\sigma^{b}(s ; t)} .
$$

The PACF $\beta(\cdot, \cdot)$ describes, for all $(t, s)$ of $\mathbb{Z}^{2}$, the partial correlation coefficient between $X(t)$ and $X(s)$ in the set $\{X(s), \ldots, X(t)\}$. For $s<t, \beta(t, s)$ is given by

$$
\overline{\beta(s, t)}=\beta(t, s)=E\left\{\eta^{f}(t ; s+1) \overline{\eta^{b}(s ; t-1)}\right\} .
$$

Furthermore, we set $\beta(t, t)=R(t, t)$ instead of 1 in order to characterize the second-order properties of the process by this function. The advantage of the PACF is its variation domain. It can be easily described in comparison with $R(\cdot, \cdot)$ which must be n.n.d. Precisely, for $t \neq s$, the magnitude of $\beta(t, s)$ is generally strictly $<1$ and if it is equal to 1 it corresponds to linear relationships; i.e. for $s<t,|\beta(t, s)|=1$ if and only if $s$ is the largest integer such that $X(t)$ belongs to $\mathcal{M}(s ; t-1)$. Our convention leads to

$$
\beta(t, s-k)=\beta(t+k, s)=0, \quad \text { for } k \geq 1 .
$$

In the same way, we have

$$
\beta(t, t-k)=\beta(t+k, t)=0, \quad \text { for } k>0
$$


when a variable $X(t)$ is almost surely equal to 0 . In both the previous situations, the process is called locally deterministic. We easily check that a process is PC of period $T$ if and only if its PACF satisfies:

$$
\beta(t+T, s+T)=\beta(t, s) \quad \text { for all }(t, s) \in \mathbb{Z}^{2} .
$$

We denote, by $\mathcal{D}_{\beta}^{T}$, the set of $T$-periodic functions which satisfy the above conditions. Then $\mathcal{D}_{\beta}^{T}$ can be parameterized by $T$ functions defined on $\mathbb{N}, \beta_{t}(n)=$ $\beta(t, t-n), t=1, \ldots, T$, which are only subject to the following conditions:

1. $\beta_{t}(0) \geq 0$ and $\left|\beta_{t}(n)\right| \leq 1, n \geq 1$,

2. $\beta_{t}(0)=0 \Rightarrow \beta_{t}(n)=\beta_{(t+n)[T]}(n)=0, n \geq 1$,

3. $\left|\beta_{t}(j)\right|=1, j>0 \Rightarrow \beta_{t}(n)=\beta_{(t+n-j)[T]}(n)=0, n>j$,

where $t[T]$ is the integer $r_{t}$ in $[1, \ldots, T]$ such that $t=q_{t} T+r_{t}$ and $q_{t} \in \mathbb{Z}$. Notice that the points (1) and (2) cater to the locally deterministic case. Otherwise, the conditions are reduced to $\beta_{t}(0)>0$ and $\left|\beta_{t}(n)\right|<1, n \geq 1$, for $t=1, \ldots, T$. On the contrary, any function in $\mathcal{D}_{\beta}^{T}$ is the PACF of a PC process. In a similar way, we denote by $\mathcal{D}_{R}^{T}$, the set of the n.n.d. $T$-periodic functions.

\subsection{Correspondence between the ACF and the PACF}

In the non-stationary case, the correspondence between $R(\cdot, \cdot)$ and $\beta(\cdot, \cdot)$, on a subset $E$ of $\mathbb{Z}^{2}$ is one-to-one if and only if $\{(u, v), s \leq v \leq u \leq t\} \subseteq E$ when $(s, t) \in E$ (see Dégerine and Lambert-Lacroix, 2003) (e.g. $\left.E=[s, \ldots, t]^{2}\right)$. This correspondence is one-to-one because $\beta(t, s)$ depends on all the values $R(u, v), s \leq v \leq u \leq t$. Reciprocally, $R(t, s)$ depends on all the values $\beta(u, v), s \leq v \leq u \leq t$. In the periodical case, because of the periodicity property of the ACF and the PACF, it is reasonable to consider domains of the form

$$
E\left(p_{1}, \ldots, p_{T}\right)=\left\{(t, s) \in \mathbb{Z}^{2}, 0 \leq t-s \leq p_{t[T]}\right\}
$$

where $p_{1}, \ldots, p_{T}$ are any positive integers. Let us point out that $(t, s) \in E\left(p_{1}, \ldots, p_{T}\right)$ if and only if $(t+T, s+T) \in E\left(p_{1}, \ldots, p_{T}\right)$. This means that the variance of $X(t)$ and the covariance, or partial correlation, between $X(t)$ and the elements of $\left\{X(t-1), \ldots, X\left(t-p_{t[T]}\right)\right\}$ are given for each $t$. For instance, it is the case for periodic autoregressive models of order $\left(p_{1}, \ldots, p_{T}\right)$ considered in Section 2.3. However, we cannot take any arbitrary integer in order to have one-to-one correspondence. We then have the following property (its proof is given in the Appendix).

Proposition 1. The correspondence between $R(\cdot, \cdot)$ and $\beta(\cdot, \cdot)$ over $E\left(p_{1}, \ldots, p_{T}\right)$ is one-to-one if and only if $p_{t} \leq p_{t-1}+1$ for $t=1, \ldots$ w with $p_{0}=p_{T}$. 
Such a constrained set will be denoted below by $E_{c}\left(p_{1}, \ldots, p_{T}\right)$. If the orders are equal, $p_{1}=p_{2}=\cdots=p_{T}=p$, then $p_{t}=p \leq p+1=p_{t-1}+1, t=1, \ldots, T$. The correspondence between $R(\cdot, \cdot)$ and $\beta(\cdot, \cdot)$ on $E_{c}\left(p_{1}, \ldots, p_{T}\right)$ is given by the periodic Levinson-Durbin (PLD) algorithm obtained by restricting the extended Levinson-Durbin algorithm of the general non-stationary case of Dégerine and Lambert-Lacroix (2003).

Sakai (1983) also proposes to characterize the autocovariance matrix sequence of a $T$-multivariate non-locally deterministic stationary process by $T$ sets of scalar normalized partial correlation coefficients. Indeed, there is a one-to-one correspondence between PC and multivariate stationary processes (see Gladysev, 1961). Precisely, let us define the $j$ th component of the $T$-multivariate process $Y(\cdot)=\{Y(t), t \in \mathbb{Z}\}$ by $Y_{j}(t)=X(j+T(t-1))$ for $j=1, \ldots, T$ and $t \in \mathbb{Z}$. Then $Y(\cdot)$ is wide-sense stationary if and only if the associated scalar process $X(\cdot)$ is PC of period $T$ (Gladysev, 1961). It follows that the autocovariance matrices $R_{k}=E\left\{Y(t+k) Y(t)^{*}\right\}$ of $Y(\cdot)$ can be expressed directly in terms of $R(\cdot, \cdot)$ :

$$
\left\{R_{k}\right\}_{i j}=R(i+k T, j) \text { for } i, j=1, \ldots, T .
$$

Sakai (1982) gives a Levinson-type circular recursive algorithm which establishes the correspondence between $R_{0}, \ldots, R_{N}$, and the sets of its normalized coefficients in the non-locally deterministic case. Indeed, this algorithm is equivalent to the PLD algorithm on $E_{c}(N T+i-1, i=1, \ldots, T)$ and these coefficients are equal to $-\beta_{t}(k)$.

\subsection{Periodic autoregressive processes}

A process $X(\cdot)$ is periodic autoregressive of period $T$ and order $\left(p_{1}, \ldots, p_{T}\right)$ $\left(\operatorname{PAR}\left(p_{1}, \ldots, p_{T}\right)\right)$ if it is $\mathrm{PC}$ of period $T$ and if for all $t \in \mathbb{Z}$, some constants $a_{t}(k)$, $k=1, \ldots, p_{t}$, exist such that

$$
\sum_{k=0}^{p_{t}} a_{t}(k) X(t-k)=\varepsilon(t), \quad a_{t}(0)=1, \quad a_{t}\left(p_{t}\right) \neq 0,
$$

where $\varepsilon(\cdot)$ is the innovation process with variance $\sigma_{\varepsilon}^{2}(\cdot)$ and $a_{t+T}(k)=a_{t}(k), k=$ $1, \ldots, p_{t}$. Note that this definition is slightly different from that of Jones and Brelsford (1967), because we suppose that $\varepsilon(\cdot)$ is the innovation process. The PAR models are easily characterized by the PACF. According to the Theorem 4 of Dégerine and Lambert-Lacroix (2003) and the properties of PC processes, a process is $\operatorname{PAR}\left(p_{1}, \ldots, p_{T}\right)$ if and only if its $\mathrm{PACF}$ is $T$-periodic and satisfies $t=$ $1, \ldots, T$ :

$$
\beta_{t}\left(p_{t}\right) \neq 0 \quad \text { and } \quad \beta_{t}(k)=0, \quad k>p_{t}
$$

The function $\beta(\cdot, \cdot)$ must be equal to zero outside the domain $E\left(p_{1}, \ldots, p_{T}\right)$. 


\subsection{The Yule-Walker extension}

We have the analogous form of the Yule-Walker equations (Pagano, 1978) for the $\operatorname{PAR}\left(p_{1}, \ldots, p_{T}\right)$ model:

$$
R(t, s)+\sum_{k=1}^{p_{t}} a_{t}(k) R(t-k, s)=\sigma_{\varepsilon}^{2}(t) \delta_{t, s}, \quad t \in \mathbb{Z}, s \leq t,
$$

where $\delta_{t, s}=1$ if $t=s$, else 0 and $a .(\cdot), \sigma_{\varepsilon}^{2}(\cdot)$ are the $T$-periodic parameters of the model. It follows that the PAR models can be parameterized equivalently either by the first autocovariance coefficients or the partial autocorrelation ones.

Let us recall the scalar stationary case $(T=1)$ where $R(0), \ldots, R(p)(R(k)=$ $R(t, t-k))$ are the coefficients of an ACF; the function $R(\cdot, \cdot)$ is given on $E_{c}(p)$. The Yule-Walker equations are used to determine the ACF of the autoregressive model of order less than $p$ associated with these first coefficients outside the domain $E_{c}(p)$. Indeed, the equations

$$
R(i)+\sum_{k=1}^{p} a(k) R(i-k)=\sigma_{\varepsilon}^{2} \delta_{i 0}, \quad i=0, \ldots, p
$$

have a unique solution $a(1), \ldots, a(p), \sigma_{\varepsilon}^{2}$, where $\sigma_{\varepsilon}^{2}>0$ in the non-locally deterministic case. The $R(\cdot)$ extension is then obtained by,

$$
R(i)=-\sum_{k=1}^{p} a(k) R(i-k), \quad i>p .
$$

The extension in terms of PACF is well adapted since it suffices to put $\beta(i)=$ $\beta(t, t-i)=0$ for $i>p$. The first coefficients $\beta(0), \ldots, \beta(p)$ can be obtained from $R(0), \ldots, R(p)$ via the Levinson-Durbin algorithm.

In the periodical case, the use of the Yule-Walker equations is more delicate. Our goal here is to explain why it does not work and give an example of this. It seems interesting to point this result out since these equations are used in the periodical case without being on the safe side. For example, Pagano (1978) proposes, for autoregressive parameter estimation, keeping the model $\operatorname{PAR}\left(p_{1}, \ldots, p_{T}\right)$ associated with the biased autocovariance estimates. This method does not always provide a solution (see Lambert-Lacroix, 2000). This problem is a consequence of the following result. The restriction to $E\left(p_{1}, \ldots, p_{T}\right)$ of an ACF $R(\cdot, \cdot)$ in $\mathcal{D}_{R}^{T}$ does not always give the first autocovariance coefficients of a PAR model of order at most $\left(p_{1}, \ldots, p_{T}\right)$ [denoted by $\left.\operatorname{PAR}\left(\leq p_{1}, \ldots, \leq p_{T}\right)\right]$. Indeed, when it is a constrained set $E_{c}\left(p_{1}, \ldots, p_{T}\right)$, the PAR model is perfectly defined as the one for which the PACF corresponds to $R(\cdot, \cdot)$ on this domain and vanishes outside. Otherwise, let $E_{c}\left(\tilde{p}_{1}, \ldots, \tilde{p}_{T}\right)$ be the lowest constrained set containing $E\left(p_{1}, \ldots, p_{T}\right)$, and let $\beta(\cdot, \cdot)$ be the PACF characterizing the possible solution. So $\beta(\cdot, \cdot)$ must vanish over $E_{c}\left(\tilde{p}_{1}, \ldots, \tilde{p}_{T}\right) \backslash E\left(p_{1}, \ldots, p_{T}\right)$. It is then possible to determine the missing values of $R(\cdot, \cdot)$ and $\beta(\cdot$,$) on E_{c}\left(\tilde{p}_{1}, \ldots, \tilde{p}_{T}\right)$. Indeed, we compute $\beta(t, s)$ with the PLD algorithm when $(t, s) \in E\left(p_{1}, \ldots, p_{T}\right)$, and otherwise $R(t, s)$. This algorithm is given 
in the Appendix. Now the $\operatorname{PAR}\left(\leq p_{1}, \ldots, \leq p_{T}\right)$ model exists if and only if $\beta(\cdot, \cdot)$ belongs to $\mathcal{D}_{\beta}^{T}$. That leads to $R(\cdot, \cdot)$ to be n.n.d. Obviously when the $\operatorname{PAR}\left(\leq p_{1}, \ldots, \leq p_{T}\right)$ does not exist, the Yule-Walker equations lead to an $R(\cdot, \cdot)$ extension which does not respect the n.n.d. property (see the example below).

On the contrary, let $\beta_{t}(k), k=0, \ldots, p_{k}, t=1, \ldots, T$, be some coefficients satisfying the $\mathcal{D}_{\beta}^{T}$ constraints. Then a $\operatorname{PAR}\left(\leq p_{1}, \ldots, \leq p_{T}\right)$ is always associated with these first coefficients. It is unique and defined by extending $\beta(\cdot, \cdot)$ to 0 beyond the model orders since this extension respects the $\mathcal{D}_{\beta}^{T}$ constraints. Clearly that shows that the use of the PACF is preferable to the ACF.

\subsection{Example}

Let $R(\cdot, \cdot)$ be the ACF defined by,

$$
\begin{gathered}
R(1,1)=1, \quad R(1,2 i+1)=(0.9)^{i+2}, \quad R(1,2 i)=(0.9)^{i} ; \\
R(2,2)=1, \quad R(2,2 i+1)=(0.9)^{i+1}, \quad R(2,2 i+2)=(0.9)^{i} \quad i \geq 1,
\end{gathered}
$$

and the remaining $R(i, j)$ are given via symmetry and periodicity. This second order structure is the one of a $\operatorname{PAR}(1,2)$ having $\operatorname{PACF} \beta(\cdot, \cdot)$ given by

$$
\begin{gathered}
\beta_{1}(0)=1, \quad \beta_{1}(1)=(0.9)^{2}, \quad \beta_{1}(k)=0, k>1 \\
\beta_{2}(0)=1, \quad \beta_{2}(1)=0.9, \quad \beta_{2}(2)=\frac{0.9 \sqrt{1-(0.9)^{2}}}{\sqrt{1-(0.9)^{4}}}, \quad \beta_{2}(k)=0, k>2 .
\end{gathered}
$$

We want to fit a $\operatorname{PAR}(0,2)$ of $\operatorname{ACF} \tilde{R}(\cdot, \cdot)$ which coincides with $R(\cdot, \cdot)$ over $E(0,2)$; i.e. $\tilde{R}(1,1)=R(1,1), \tilde{R}(2,2)=R(2,2), \tilde{R}(2,1)=R(2,1)$ and $\tilde{R}(2,0)=R(2,0)$. The domain $E_{c}(1,2)$ is the lowest constrained set containing $E(0,2)$ and Algorithm 1 (see Appendix) leads to

$$
\begin{gathered}
\tilde{\beta}_{1}(0)=1, \quad \tilde{\beta}_{1}(1)=0 \\
\tilde{\beta}_{2}(0)=1, \quad \tilde{\beta}_{2}(1)=0.9, \quad \tilde{\beta}_{2}(2)=\frac{0.9}{\sqrt{1-(0.9)^{2}}} .
\end{gathered}
$$

So, $\operatorname{PAR}(0,2)$ model having ACF given by $R(\cdot, \cdot)$ over $E(0,2)$, do not exist since $\tilde{\beta}_{2}(2)=2.0647>1$. On the contrary, the extension through the Yule-Walker equation leads to $\tilde{R}(1,0)=\tilde{R}(0,1)=0$ and

$$
R_{2,4}=\left(\begin{array}{ccc}
1 & 0 & 0.9 \\
0 & 1 & 0.9 \\
0.9 & 0.9 & 1
\end{array}\right) \text {. }
$$


Since $\operatorname{det}\left(R_{2,4}\right)=1-2 \times 0.9^{2}<0$, the Yule-Walker extension does not define an ACF. This is so every time the PAR model does not exist.

\section{EXTENSION OF AUTOCOVARIANCE COEFFICIENTS SEQUENCE}

\subsection{The extension problem}

Analogous to the stationary case, the first option (see Zhang, 1997) is to consider the second-order structure of the sequences $X(t), \ldots, X\left(t-p_{t}\right), t=1, \ldots, T$, with $p_{t} \geq 0$ which are not necessarily the same. Indeed, the $T$-periodic function $R(\cdot, \cdot)$ is known; it is defined on the set

$$
\Delta\left(p_{1}, \ldots, p_{T}\right)=\bigcup_{t=1}^{T}\left\{(u, v) \in \mathbb{Z}^{2}: t-p_{t} \leq u, v \leq t\right\} .
$$

This domain is equal to $E_{c}\left(\tilde{p}_{1}, \ldots, \tilde{p}_{T}\right)$, the lowest constrained domain containing $E\left(p_{1}, \ldots, p_{T}\right)$. Let us consider the case where $T=2, p_{1}=1$ and $p_{2}=3$. The only point which belongs to $\Delta(1,3) \backslash E(1,3)$ is $(3,1)$ and $\Delta(1,3)=E_{c}(2,3)$ (the lowest constrained set containing $E(1,3))$. The solution maximizing the MEM is a PAR model of order at most $\left(\tilde{p}_{1}, \ldots, \tilde{p}_{T}\right)$ but not $\left(p_{1}, \ldots, p_{T}\right)$. According to this remark, it is reasonable to consider directly the constrained set $E_{c}\left(p_{1}, \ldots, p_{T}\right)$.

In this situation, the PACF allows the resolution of this problem even in the locally deterministic case. With the PLD algorithm, we obtain the coefficients $\beta_{t}(n), t=1, \ldots, T, n=0, \ldots, p_{t}$, from the values of $R(\cdot ;)$ on $E_{c}\left(p_{1}, \ldots, p_{T}\right)$. So these previous values are those pertaining to ACF if and only if the quantities $\beta_{t}(n)$ satisfy the constraints of $\mathcal{D}_{\beta}^{T}$. Indeed, the correspondence between $R(\cdot, \cdot)$ and $\beta(\cdot, \cdot)$ is one-to-one on such a domain. When the coefficients $R(t, s)$ represent some ACF values, all the extensions are described through the extensions of $\beta_{t}(n)$ remaining in $\mathcal{D}_{\beta}^{T}$. However, in the locally deterministic case, we must verify that $R(\cdot, \cdot)$ satisfies the constraints related to the finite-order singularities. For example, if $\left|\beta_{i}(k)\right|=1$, the PLD algorithm gives

$$
\sigma_{i}^{f}(k+n)=\sigma_{(i+n)[T]}^{b}(k+n)=0, \quad n \geq 0,
$$

and the convention $0^{-1}=0$ (see Dégerine and Lambert-Lacroix, 2003) leads to

$$
\beta_{i}(k+n+1)=\beta_{(i+n+1)[T]}(k+n+1)=0, \quad n \geq 0 .
$$

Then the coefficients $\beta_{t}(n)$ always satisfy the $\mathcal{D}_{\beta}^{T}$ constraints related to the singularities. In this case, we must verify that the values $R(t, s),(t, s) \in E_{c}\left(p_{1}, \ldots, p_{T}\right)$, are associated with $\beta_{t}(n)$, i.e. for $l=i,(i+n+1)[T]$ :

$$
R(l, l-k-n-1)+\sum_{j=1}^{k+n} a_{l}^{f}(k+n, j) R(l-j, l-k-n-1)=0 .
$$


Notice that the extension problem considered here is more general than the one of $R_{0}, \ldots, R_{p}$, in the multivariate stationary case. It coincides with it only when $p_{i}=$ $p T+i-1, i=1, \ldots, T$.

The problem is more difficult when the domain is not constrained. Indeed, we can use the Algorithm 1 to check if there exists a $\operatorname{PAR}\left(\leq p_{1}, \ldots, \leq p_{T}\right)$. But answering in the negative does not imply that the coefficients $R(t, s)$ are not the one of ACF (as in the example of Section 2.5). To conclude, we should be able to try all the possible combinations of the PACF coefficients over $E_{c}\left(\tilde{p}_{1}, \ldots, \tilde{p}_{T}\right) \backslash E\left(p_{1}, \ldots, p_{T}\right)$ in the Algorithm 1, which is clearly impossible in practice.

\subsection{The maximum entropy method}

In the non-locally deterministic stationary case, the MEM was introduced by Burg (1967) in the following way. Let $R(0), \ldots, R(p)$ be some values of the ACF of a stationary process. The MEM consists in finding the spectral density $f$ that satisfies

$$
\int_{-\pi}^{\pi} f(\lambda) \mathrm{e}^{\mathrm{i} k \lambda} \mathrm{d} \lambda=R(k), \quad k=0, \ldots, p
$$

and maximizes

$$
\int_{-\pi}^{\pi} \ln \operatorname{det} f(\lambda) \mathrm{d} \lambda=2 \pi \ln \left[\frac{\sigma_{\varepsilon}^{2}}{2 \pi}\right],
$$

where $\sigma_{\varepsilon}^{2}$ is the innovation process variance. So this method is equivalent to choosing the process with maximum variance $\sigma_{\varepsilon}^{2}$ and the solution is the autoregressive model associated with $R(0), \ldots, R(p)$. In the non-stationary case, we have a relation between the variances $\sigma_{\varepsilon}^{2}(t)$ and the PACF (see Dégerine and Lambert-Lacroix, 2003) which leads to

$$
\sigma_{\varepsilon}^{2}(t)=\beta_{k}(0) \prod_{n=1}^{+\infty}\left[1-\left|\beta_{k}(n)\right|^{2}\right], \quad k=t[T],
$$

for PC processes of period $T$. In the non-locally deterministic case, no relationships exist between these $T$ variances. We can maximize each of the variances $\sigma_{\varepsilon}^{2}(k), k=1, \ldots, T$, separately. Furthermore, we have,

$$
\sigma_{\varepsilon}^{2}(k) \leq \beta_{k}(0) \prod_{n=1}^{p_{k}}\left[1-\left|\beta_{k}(n)\right|^{2}\right],
$$

with equality if and only if $\beta_{k}(n)=0$ for $n>p_{k}$. So the solution is the model PAR $\left(\leq p_{1}, \ldots, \leq p_{T}\right)$ associated with the values $\beta_{k}(n)$. In the locally deterministic case, some of the variances $\sigma_{\varepsilon}^{2}(k)$ are equal to 0 and the PACF must vanish in places. Thus, the same approach can be used but only for the variances not already equal to 0 . Then the solution is again the PAR model. 
It is easy to see that the functions $\beta_{t}(\cdot), t=1, \ldots, T$, are a set of PACF of secondorder stationary processes [i.e. for fixed $t, \beta_{t}(\cdot) \in \mathcal{D}_{\beta}^{1}$ ]. Let $Y_{t}(\cdot)$, for $t=1, \ldots, T$, be a stationary process with second-order structure given by $\beta_{t}(\cdot)$. The stationary process $Y_{t}(\cdot)$ has the same variance as the variables $X(t)$. Furthermore, the partial correlation between $Y_{t}(s)$ and $Y_{t}(s-k)$ in the set $\left\{Y_{t}(s-k), \ldots, Y_{t}(s)\right\}, s \in \mathbb{Z}, k=0,1, \ldots$, is given by the partial correlation between $X(t)$ and $X(t-k)$ in the set $\{X(t-k), \ldots, X(t)\}$. So our MEM extension deals with maximizing the entropy of each stationary vector $Y_{t}(1), \ldots, Y_{t}\left(p_{t}\right), t=1, \ldots, T$ of second-order structure given by $\beta_{t}(0), \ldots, \beta_{t}\left(p_{t}\right)$.

\subsection{Comparison with other approaches}

Zhang (1997) considers a $T$-periodic function $R(\cdot, \cdot)$ defined on the set $\Delta\left(p_{1}, \ldots, p_{T}\right)$ (described in Section 3.1) with any positive integers $p_{1}, \ldots, p_{T}$, such that $R_{t-p_{t}: t}$ is positive definite for all $t=1, \ldots, T$. Next, the author claims that there is a Gaussian $\operatorname{PAR}\left(\leq p_{1}, \ldots, \leq p_{t}\right)$ (see proof of the Theorem 1) which covariance structure coincides with $R(\cdot$,$) over \Delta\left(p_{1}, \ldots, p_{t}\right)$. Let us recall that this domain is equal to the lowest constrained domain $E_{c}\left(\tilde{p}_{1}, \ldots, \tilde{p}_{T}\right)$ containing $E\left(p_{1}, \ldots, p_{T}\right)$. When the orders $\left(\tilde{p}_{1}, \ldots, \tilde{p}_{T}\right)$ differ from $\left(p_{1}, \ldots, p_{t}\right)$, this approach is incorrect. First, as in Section 2.4 , there does not always exist a $\operatorname{PAR}\left(\leq p_{1}, \ldots, \leq p_{t}\right)$. Second, even if the PAR exists, there is no reason that its second-order structure is given by $R(\cdot, \cdot)$ over $\Delta\left(p_{1}, \ldots, p_{t}\right) \backslash E\left(p_{1}, \ldots, p_{t}\right)$. We can only claim that there exists a PAR of order $\left(\leq \tilde{p}_{1}, \ldots, \leq \tilde{p}_{t}\right)$ but not $\left(\leq p_{1}, \ldots, \leq p_{t}\right)$. Consequently, the domain $\Delta\left(p_{1}, \ldots, p_{t}\right)$ seems to be ill defined.

In Alpay et al. (2001), another approach based on the cyclo-correlation functions is used to treat an extension problem similar to ours. Indeed, from the periodicity in $t$ of $R(t+n, t)$, Gladysev (1961) introduces the cyclo-correlation functions $B_{k}(n), k=0, \ldots, T-1$, of the representation

$$
R(t+n, t)=\sum_{k=0}^{T-1} B_{k}(n) \mathrm{e}^{\frac{2 \pi i k t}{T}}, \quad(t, n) \in \mathbb{Z}^{2} .
$$

The inverse Fourier transform leads to

$$
B_{k}(n)=\frac{1}{T} \sum_{t=0}^{T-1} R(t+n, t) \mathrm{e}^{-\frac{2 \pi i k t}{T}}, \quad n \in \mathbb{Z} .
$$

Now these functions characterize an element $R(\cdot, \cdot)$ of $\mathcal{D}_{R}^{T}$ if and only if the matrices $B(n), n \geq 0$, defined by

$$
B(n)_{l, j}=B_{j-l}(n) \mathrm{e}^{-\frac{2 \pi i l n}{T}}, \quad l, j=0, \ldots, T-1,
$$

where $B_{-k}(n)=B_{T-k}(n), k=1, \ldots, T-1$, are the autocovariance matrices of some $T$-dimensional stationary random process. In Alpay et al. (2001), the authors have considered the extension problem of such functions from the data $B_{k}(n), k=$ 
$0, \ldots, T-1,0 \leq n \leq N$, and in the non-locally deterministic case. Then the whole extension of such data is described through the $B(n)$ values respecting eqn (1). The problem is shifted to a Caratheodory-Fejer problem with symmetry constraints. This procedure consists in extending a particular sequence of autocorrelation matrices. They find again the PAR model as solution of the MEM. Clearly, this approach is more delicate that the one presented here. On the contrary, this problem is equivalent to the one presented in the Section 3.1 with the particular set $E_{c}(N, \ldots, N)$ and the use of the PACF allows to resolve this one, even in the degenerate case.

\section{APPENDIX}

PROOF OF THE PROPOSITION 1

In the non-stationary case, the correspondence between $R(\cdot, \cdot)$ and $\beta(\cdot, \cdot)$ on a subset $E$ of $\mathbb{Z}^{2}$ is one-to-one if and only if $\{(u, v), s \leq v \leq u \leq t\} \subseteq E$ when $(s, t) \in E$ (see Dégerine and Lambert-Lacroix, 2003). Let $E\left(p_{1}, \ldots, p_{T}\right)$ be a domain for which the correspondence between $R(\cdot, \cdot)$ and $\beta(\cdot$,$) is one-to-one. For t=1, \ldots, T, \beta\left(t, t-p_{t}\right)$ depends on the value $R\left(t-1, t-p_{t}\right)$ and the point $\left(t-1, t-p_{t}\right)$ must belong to $E\left(p_{1}, \ldots, p_{T}\right)$; i.e. $p_{t-1} \geq t-1-\left(t-p_{t}\right)=p_{t}-1 \quad$ (with $\left.p_{0}=p_{T}\right)$. Conversely, we must show that if $E\left(p_{1}, \ldots, p_{T}\right)$ is a set with $p_{t} \leq p_{t-1}+1$ for $t=1, \ldots, T$ (with $\left.p_{0}=p_{T}\right)$, then it is a domain of one-to-one correspondence. We propose a proof by contradiction. Let us suppose that there exists $t$ such that $p_{t}>p_{t-1}+1$ and that the set $E\left(p_{1}, \ldots, p_{T}\right)$ is a domain of one-to-one correspondence. Since $p_{t}>p_{t-1}+1$, we have $t-1-\left(t-p_{t}\right)=p_{t}-1>p_{t-1}$ and the point $\left(t-1, t-p_{t}\right)$ does not belong to $E\left(p_{1}, \ldots, p_{T}\right)$. On the contrary, since the set $E\left(p_{1}, \ldots, p_{T}\right)$ is a domain of one-to-one correspondence, we can show as above that the point $\left(t-1, t-p_{t}\right)$ belongs to $E\left(p_{1}, \ldots, p_{T}\right)$. That leads to a contradiction.

\section{ALGORITHM}

The input variables for the following procedure are given by $\left(p_{1}, \ldots, p_{T}\right)$ and

$$
R_{k}(n)(=R(k, k-n)), \quad k=1, \ldots, T, \quad n=0, \ldots, p_{k} .
$$

\section{Algorithm 1}

1 Computation of $\left(\tilde{p}_{1}, \ldots, \tilde{p}_{T}\right)$ step

$$
\begin{aligned}
& k_{\max } \leftarrow \operatorname{argmax}\left\{p_{k}, k=1, \ldots, T\right\} \\
& \text { indice } \leftarrow\left(k_{\max }, k_{\max }-1, \ldots, 1, T, T-1, \ldots, k_{\max }+1\right) \\
& \tilde{p}_{\text {indice }[1]} \leftarrow p_{\text {indice }[1]} \\
& \text { for }(k=2: T) \\
& \tilde{p}_{\text {indice }[k]} \leftarrow \max \left(p_{\text {indice }[k-1]}-1, p_{\text {indice }[k]}\right) \\
& \text { endfor }(k=2: T)
\end{aligned}
$$


2 Computation of $\beta_{k}(n), k=1, \ldots, T, n=0, \ldots, \tilde{p}_{k}$ Step

for $(k=1: T)$ :

$$
\begin{aligned}
\beta_{k}(0) & \leftarrow R_{k}(0) \\
\sigma_{k}^{f 2}(0) & \leftarrow R_{k}(0) \\
\sigma_{k}^{b 2}(0) & \leftarrow R_{k}(0)
\end{aligned}
$$

endfor $(k=1: T)$

for $\left(n=1: p_{k_{\max }}\right)\left(\right.$ with the conventions $\sum_{j=1}^{0} \ldots=0$ and $\left.0^{-1}=0\right)$

for $(k=1: T)$

if $\left(n \leq p_{k}\right)$ and $\left(n \leq \tilde{p}_{k}\right)$

$$
\beta_{k}(n) \leftarrow \frac{R_{k}(n)+\sum_{j=1}^{n-1} a_{k}^{f}(n-1, j) R_{(k-j)[T]}(n-j)}{\sigma_{k}^{f}(n-1) \sigma_{(k-1)[T]}^{b}(n-1)}
$$

endif $\left(n \leq p_{k}\right)$ and $\left(n \leq \tilde{p}_{k}\right)$

if $\left(n>p_{k}\right)$ and $\left(n \leq \tilde{p}_{k}\right)$

$$
\begin{aligned}
& \beta_{k}(n) \leftarrow 0 \\
& R_{k}(n) \leftarrow-\sum_{j=1}^{n-1} a_{k}^{f}(n-1, j) R_{(k-j)[T]}(n-j)
\end{aligned}
$$

endif $\left(n>p_{k}\right)$ and $\left(n \leq \tilde{p}_{k}\right)$

if $\left(n \leq \tilde{p}_{k}\right)$

$$
\begin{aligned}
\sigma_{k}^{f 2}(n) & \leftarrow\left[1-\left|\beta_{k}(n)\right|^{2}\right] \sigma_{k}^{f 2}(n-1) \\
\sigma_{k}^{b 2}(n) & \leftarrow\left[1-\left|\beta_{k}(n)\right|^{2}\right] \sigma_{(k-1)[T]}^{b 2}(n-1) \\
a_{k}^{f}(n, n) & \leftarrow-\beta_{k}(n) \frac{\sigma_{k}^{f}(n-1)}{\sigma_{(k-1)[T]}^{b}(n-1)} \\
a_{k}^{b}(n, n) & \leftarrow-\overline{\beta_{k}(n)} \frac{\sigma_{(k-1)[T]}^{b}(n-1)}{\sigma_{k}^{f}(n-1)}
\end{aligned}
$$

for $(j=1: n-1)$

$$
\begin{aligned}
& a_{k}^{f}(n, j) \leftarrow a_{k}^{f}(n-1, j)+a_{k}^{f}(n, n) a_{(k-1)[T]}^{b}(n-1, n-j) \\
& a_{k}^{b}(n, j) \leftarrow a_{(k-1)[T]}^{b}(n-1, j)+a_{k}^{b}(n, n) a_{k}^{f}(n-1, n-j)
\end{aligned}
$$

endfor $(j=1: n-1)$

endif $\left(n \leq \tilde{p}_{k}\right)$

endfor $(k=1: T)$

endfor $\left(n=1: p_{k_{\max }}\right)$

\section{Return}

$$
\beta_{k}(n), \quad k=1, \ldots, T, n=0, \ldots, p_{k} .
$$

(c) Blackwell Publishing Ltd 2005 
The author would like to thank the referee for useful comments which improve significantly the presentation of the paper.

NOTES

Corresponding author: Sophie Lambert-Lacroix, LMC-IMAG, BP 53, 38041 Grenoble Cedex 9, France. E-mail: sophie.lambert@imag.fr

\section{REFERENCES}

Alpay, D., Chevreuil, A. and Loubaton, P. (2001) An extension problem for discrete-time periodically correlated stochastic processes. J. Time Ser. Anal. 22, 1-11.

Boshnakov, G. (1994) Periodically correlated sequences:some properties and recursions. Research Report 1, Division of Quality Technology and Statistics, Luleo University, Sweden.

BurG, J. P. (1967) Maximum entropy spectral analysis. 37th Annual International S.E.G. Meeting, Oklahoma City.

BurG, J. P. (1968) A new analysis technique for time series data. NATO Advanced Study Institute on Signal Processing, Enschede, Netherlands.

Constantinescu, T. (1996) Schur Parameters, Factorization and Dilatation Problems. Basel: Birkhauser Verlag.

DÉGerine, S. (1993) Sample partial autocorrelation function. IEEE Trans. Signal Process. 41, 403-7.

DÉgerine, S. and Lambert-Lacroix, S. (2003) Partial autocorrelation function of a nonstationary time series. J. Multivariate Anal. 87, 46-59.

GARDNER, W. A. (1994) An Introduction to cyclostationary signals. In Cyclostationarity in Communications and Signal Processing (ed. W.A. GARDner), Chapter 1. New York: IEEE Press, pp. $1-90$.

Gladysev, E. G. (1961) Periodically random sequences. Soviet Mathematics, 2, 385-8.

Jones, R. H. and Brelsford, W. M. (1967) Time series with periodic structure. Biometrika 54, 403-8.

LAmbert-Lacroix, S. (2000) On periodic autoregressive processes estimation. IEEE Trans. Signal Process. SP-48, 1800-3.

Pagano, M. (1978) On periodic and multiple autoregressions. Ann. Statist. 6, 1310-7.

Ramsey, F. L. (1974) Characterization of the partial autocorrelation function. Ann. Statist. 2, 1296301.

SAKai, H. (1982) Circular lattice filtering using Pagano's method. IEEE Trans. Acoust., Speech Signal Process. ASSP-30, 279-87.

SAKAI, H. (1983) Covariance matrices characterization by a set of scalar partial autocorrelation coefficients. Ann. Statist. 11, 337-40.

Yule, G. U. (1907) On the theory of correlation for any number of variables, treated by a new system of notation. R. Soc. Proc. 79, 85-96.

ZhAng, H. (1997) Maximum entropy modeling of periodically correlated processes. IEEE Trans. Inform. Theory IT-43, 2033-5. 